Elsevier Editorial System(tm) for International Journal of Production Economics Manuscript Draft

Manuscript Number:

Title: Lot Sizing versus Batching in the Production and Distribution Planning of Perishable Goods

Article Type: Full Length Article

Keywords: Integrated Planning; Lot Sizing and Scheduling; Batching; Vehicle Routing Problem with Time Windows; Perishability

Corresponding Author: Dr. Pedro Sanches Amorim, PhD

Corresponding Author's Institution: Universidade do Porto, Faculdade de Engenharia

First Author: Pedro Sanches Amorim, PhD

Order of Authors: Pedro Sanches Amorim, PhD; Márcio Belo, MSc; Franklina Toledo, PhD; Christian

Almeder, PhD; Bernardo Almada-Lobo, PhD

Abstract: Joint production and distribution planning at the operational level has received a great deal of attention from researchers. In most industries these processes are decoupled by means of final goods inventory that allow for a separated planning of these tasks. However, for example, in the catering industry, an integrated planning framework tends to be more favorable due to the perishable nature of the products that forces a make-to-order production strategy. So far this planning problem has only been addressed by allowing the batching of orders. The main contribution of this paper is to extend this approach and prove the importance of lot sizing for make-to-order systems when perishability is explicitly considered. The value of considering lot sizing versus batching is further investigated per type of production scenario. Overall, results point that lot sizing is able to deliver better solutions than batching. The added flexibility of lot sizing allows for a reduction on production setup costs and both fixed and variable distribution costs. Finally, the savings derived from lot sizing are leveraged by customer oriented time windows and production systems with non-triangular setups. 


\section{“Lot Sizing versus Batching in the Production and Distribution Planning of Perishable Goods"}

Pedro Amorim, Márcio Belo, Franklina Toledo, Christian Almeder and Bernardo Almada-Lobo

Joint production and distribution planning at the operational level has received a great deal of attention from researchers. In most industries these processes are decoupled by means of final goods inventory that allow for a separated planning of these tasks. However, for example, in the catering industry, an integrated planning framework tends to be more favorable due to the perishable nature of the products that forces a make-to-order production strategy. So far this planning problem has only been addressed by allowing the batching of orders.

Therefore, the major contribution of this paper is to evaluate whether lot sizing decisions may deliver better results than batching when this integrated problem tackles perishability. After proving that lot sizing should be considered in this problem setting, the secondary contribution is to understand the conditions that leverage the benefits of lot sizing versus batching.

The logistic setting of our operational problem encompasses multiple perishable products subject to sequence dependent changeovers, which have to be delivered in a certain route by one of the available vehicles. We have developed models for accurately integrate both lot sizing and batching with the vehicle routing problem with time windows.

Computational results show that lot sizing is able to decrease the integrated production and distribution costs on very different types of instances. Both customer oriented time windows and production environments with non-triangular setups seem to favour the importance of considering lot sizing in this operational problem. In fact, the lot sizing solution could achieve a better performance by: reducing the number of setups, changing the sequence, reducing setup costs, reducing the number of vehicles and/or the total travelled distance. 


\title{
Lot Sizing versus Batching in the Production and Distribution Planning of Perishable Goods
}

\author{
P. Amorim ${ }^{\mathrm{a}, *}$, M. Belo ${ }^{\mathrm{b}}$, F.M.B. Toledo ${ }^{\mathrm{b}}$, C. Almeder ${ }^{\mathrm{c}}$, B. Almada-Lobo ${ }^{\mathrm{a}}$ \\ ${ }^{a}$ INEC TEC, Faculdade de Engenharia, Universidade do Porto, Rua Dr. Roberto Frias, \\ $s / n, 4600-001$ Porto, Portugal \\ ${ }^{b}$ Instituto de Ciências Matemáticas e de Computação, Universidade de São Paulo, Av. \\ do Trabalhador São-Carlense, 400, CP. 668, São Carlos, SP 13560-970, Brazil \\ ${ }^{c}$ Department of Supply Chain Management, European University Viadrina Frankfurt \\ (Oder), Große Scharrnstr. 59, 15230 Frankfurt (Oder), Germany
}

\begin{abstract}
Joint production and distribution planning at the operational level has received a great deal of attention from researchers. In most industries these processes are decoupled by means of final goods inventory that allow for a separated planning of these tasks. However, for example, in the catering industry, an integrated planning framework tends to be more favorable due to the perishable nature of the products that forces a make-to-order production strategy. So far this planning problem has only been addressed by allowing the batching of orders. The main contribution of this paper is to extend this approach and prove the importance of lot sizing for make-to-order systems when perishability is explicitly considered. The value of considering lot sizing versus batching is further investigated per type of production scenario. Overall, results point that lot sizing is able to deliver better solutions than batching. The added flexibility of lot sizing allows for a reduction on production setup costs and both fixed and variable distribution costs. Finally, the savings derived from lot sizing are leveraged by customer oriented time windows and production systems with non-triangular setups.
\end{abstract}

Keywords: Integrated Planning, Lot Sizing and Scheduling, Batching,

*Corresponding author

Email addresses: amorim.pedro@fe.up.pt (P. Amorim), marciobf@icmc.usp.br (M. Belo), fran@icmc.usp.br (F.M.B. Toledo), almeder@europa-uni.de (C. Almeder), almada.lobo@fe.up.pt (B. Almada-Lobo) 
Vehicle Routing Problem with Time Windows, Perishability 
1

2

3

\section{Introduction}

Strategic, tactical and operational integration of the production and distribution processes is reported as being able to deliver better results for companies than a decoupled approach (Park, 2005; Sarmiento \& Nagi, 1999). Very often this integration is driven by a management decision, rather than by an actual need of the underlying processes. However, when the final products are not allowed to be stocked due to, for example, freshness reasons this integration scenario becomes imperative. Within these three decision levels, it is on the operational one where more research needs to be conducted (Chen, 2009), since actual models fail to be accurate and detailed enough for the real-world problems.

The motivation for studying the operational integrated production and distribution problem comes from very practical industry situations when it is not possible or advisable to keep final inventory decoupling these two processes. In this case, companies are forced to engage in a make-to-order production strategy. Therefore, the production for a certain demand order may only start after the order arrival. The examples found in practice are related to the computer assembly industries, the food-catering, the industrial adhesive materials or the ready-mixed concrete. The importance of a holistic vision of these processes is driven by very demanding customers requiring a product that cannot wait a long time to be delivered after production. These products, having a very short lifespan, will be called hereafter as perishable. Hence, the considered operational integrated production and distribution problem relates to the decisions on how to serve a set of customers with demand for different products. The planner has to simultaneously decide on the production planning and vehicle routing, in a setting where inventory is not allowed (i.e. no inventory is carried from one planning horizon to the subsequent).

Regarding the production process, the definitions proposed by Potts \& Van Wassenhove (1992) are followed, where batching is defined as the decision of whether or not to schedule similar jobs contiguously and lot sizing refers to the decision of when and how to split a production lot of identical items into sublots. Note that processing times are proportional to the quantities processed in both cases. The modelling of our problem considers a complex production system that is accurately synchronized with the distribution process to allow for as much flexibility as possible. Therefore, no specific industry constraints are modelled, but instead the formulation is as general 
as possible. Several parallel production lines with sequence dependent setups are taken into account. Moreover, the demand from different customers for a set of products has to be delivered within strict time windows on different routes that have to be determined together with the production planning.

So far the research community has tackled this operational integrated production and distribution problem by batching orders of customers as if lot sizing decisions were never to yield a better solution. This is clearly not the case in the production planning literature where the importance of considering lot sizing and scheduling simultaneously is consensual for the multi-period setting (for example Almada-Lobo et al., 2010). To the best of our knowledge, the incorporation of lot sizing decisions in the operational production and distribution problem has never been analysed. Therefore, a major contribution of this paper is to evaluate whether lot sizing decisions may deliver better results than batching when this integrated problem tackles perishability. After proving that lot sizing should be considered in this problem setting, the secondary contribution is to understand the conditions that leverage the benefits of lot sizing versus batching.

The remainder of this paper is organized as follows. The next section reviews the literature on the operational integrated production and distribution problem. Section 3 describes the considered problem and proposes two mathematical formulations for the operational production and distribution problem of perishable goods: one considering batching and the other lot sizing. In Section 4, the results of the computational study are presented and the impact of considering lot sizing versus batching is assessed. Finally, the paper is concluded in Section 5 with the main findings and ideas for future work.

\section{Literature Review}

The literature in integrated production and distribution problems is vast and, therefore, only the papers very related to the scope of this work will be reviewed here. Our problem statement refers to the gap pointed out, in the review of Chen (2009), about operational integrated models dealing with multi-customer batch delivery problems with routing.

The research community has tackled this integrated production and distribution problem by batching orders in the production process. In Chen \& Vairaktarakis (2005) orders are delivered right after their production completion time. The authors model a single product to be scheduled on the 
production line(s) and an unlimited number of vehicles, with a fixed capacity, which perform the routing. This work also investigates the value of integration, comparing the use of a decoupled versus an integrated approach. They conclude that the improvement is more significant when the goal is to minimize the average delivery time than the maximum delivery time. In Geismar et al. (2008) product perishability is taken into account and there is a single production facility with a constant production rate. The routing process is performed by a single, capacitated vehicle that may return to the facility, therefore, performing multiple trips during the planning period. The objective is to determine the minimum makespan of the integrated production and distribution for a given set of customers. Armstrong et al. (2007) solve a related problem with a single product subject to a fixed lifespan that is also delivered by a single vehicle, but, in this case, there is no possibility of performing multiple trips. Moreover, the sequence of production and distribution is fixed and forced to be the same. Finally, Chen et al. (2009) present a model that considers stochastic demand for multiple products subject to perishability. The production environment does not consider setups between products and the delivery function is assured by a set of capacitated vehicles, however, the vehicle operating costs are disregarded.

Again, none of the aforementioned papers on the operational integrated production and distribution planning include lot sizing decisions. However, on pure production scheduling, the advantages of lot sizing over batching for a leaner environment have been proven. Santos \& Magazine (1985); Wagner \& Ragatz (1994); Low \& Yeh (2008) show how lot sizing can reduce lead time in the scheduling of machines. Moreover the impact of setup times is investigated. Nieuwenhuyse \& Vandaele (2006) proves that lot sizing improves the reliability of the deliveries in a system accounting for production and direct deliveries to customers.

Based on this literature review the contribution of this paper is clearer. Firstly, it investigates the potential performance improvement that lot sizing decisions may add to the operational production and distribution planning (in relation to only batching orders). Secondly, previous studies are extended by considering a more general production system with sequence-dependent costs and times between products. 


\section{Problem Statement and Mathematical Formulations}

In this section, the problem statement is given as well as two mathematical formulations for this problem. The first formulation models the operational integrated production and distribution problem that only considers batching of orders (I-BS-VRPTW) and the second formulation extends the first one by considering the sizing of the lots (I-LS-VRPTW). Both models are then compared.

The operational integrated production and distribution planning problem considered in this work consists of a set $M$ of parallel lines $l=1, \ldots, m$ with limited capacity that produce a set $P$ of items (or products) $i, j=1, \ldots, p$ to be delivered to a set $N$ of customers $c, d=1, \ldots, n$ through a set $A$ of $\operatorname{arcs}$ $(c, d)$. The delivery is assured by a set $K$ of identical fixed capacity vehicles indexed by $k=1, \ldots, o$ initially located at a depot. Hence, the routing can be defined on a directed graph $G=(V, A), V=N \cup\{0, n+1\}$, where the depot is simultaneously represented by the two vertices 0 and $n+1$, and, therefore, $|V|=n+2$.

Some of the products may be perishable while others last substantially beyond the considered planning horizon. Furthermore, the utilization of equipment, such as ovens in the food-catering, makes the changeover between different products dependent on the sequence. Hence, products are to be scheduled on the parallel production lines over a finite planning horizon that ranges up to the time of the last scheduled delivery.

The distribution is performed using several vehicles serving multiple customers on different routes. There exists a variable cost dependent on the total distance travelled and a fixed cost for each vehicle used. It is assumed that there are no fleet constraints such that any distribution plan can be executed. This assumption is realistic since reference contracts are usually established assuring that there always exists a fleet of sufficient size available. The two models determine the routing taking into account the vehicle capacity, and the time and cost to travel from one customer/depot to another. A customer order may aggregate several products that have to be delivered within strict time windows with a single delivery (i.e., split deliveries are not allowed). Moreover, it is assumed that demand is deterministic.

The challenge is to model the production and distribution problem that minimizes total cost of the supply chain covering these processes over the short planning horizon.

The main advantage of these models comes from the accurate synchro- 
nization of the two planning processes. While at the tactical level, the integrated production and distribution planning has the possibility to assume that at the end of the period, after production, one will start the delivery process to all customers, this assumption is not possible at the operational level. At this level one needs to go one step further and be sure that the production times of the customer orders are accurately traced so that as soon as a customer has his order completed, the vehicle servicing him may depart. However, the departure only takes place after the last customer's order (serviced by the same vehicle) has been produced.

Consider the following indices, parameters and decisions variables needed to formulate the operational production (with batching or lot sizing decisions) and distribution models.

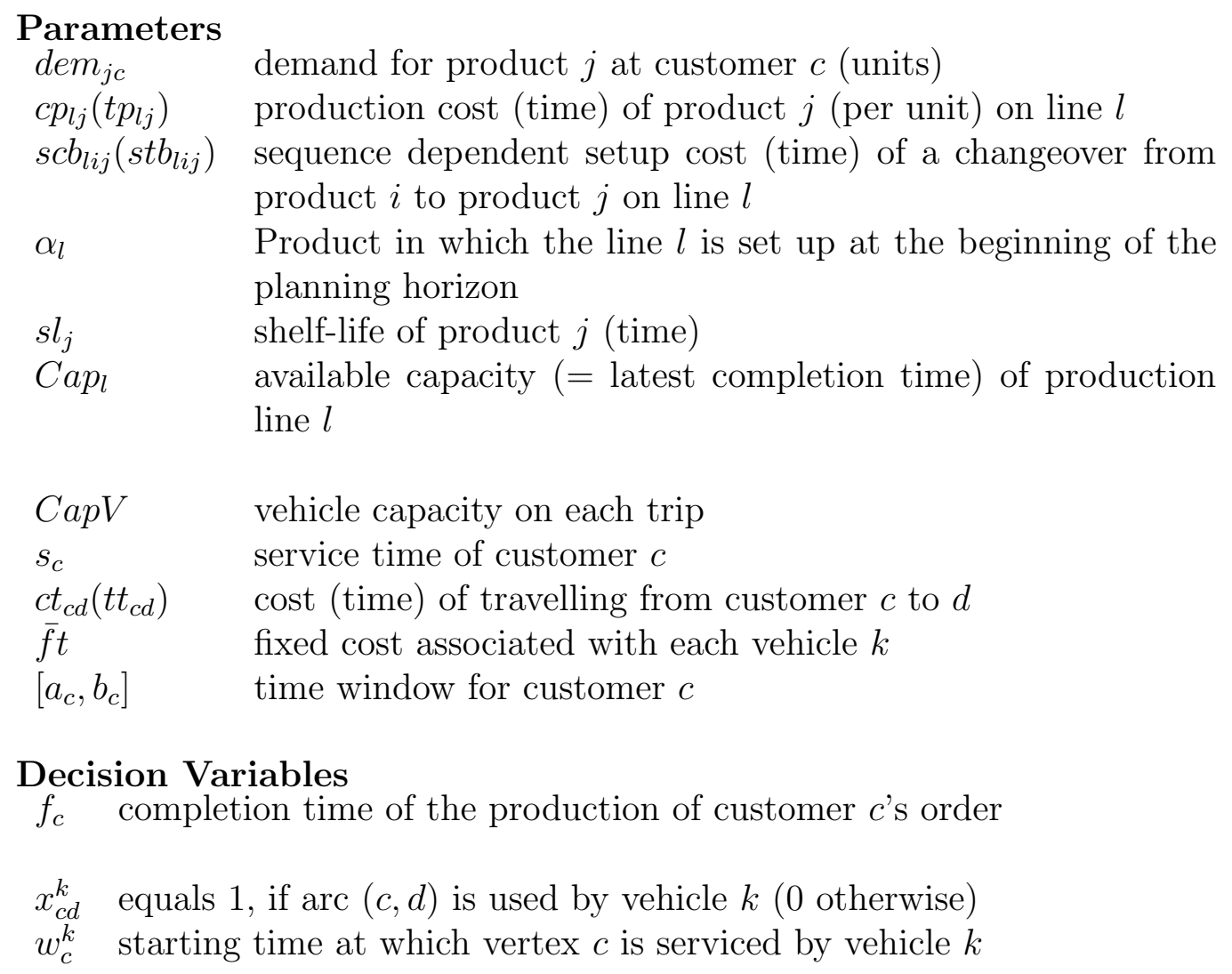




\subsection{Integrated Batch Scheduling and Vehicle Routing Problem (I-BS-VRPTW)}

This production planning modelling of this formulation is based on the work of Mendez et al. (2000). A job is given by each pair product-customer $(j, c)$ with positive demand. Let $H$ denote the set of these jobs $(H=$ $\left.\left\{(j, c), j \in P, c \in N \mid \operatorname{dem}_{j c}>0\right\}\right)$.

In order to formulate the integrated problem considering batching decisions, the following additional decision variables are needed to be added to the aforementioned ones.

\section{Decision Variables}

$R_{l(j, c)} \quad$ equals 1 , if job $(j, c)$ is produced on line $l$ ( 0 otherwise)

$R 0_{l(j, c)} \quad$ equals 1 , if job $(j, c)$ is the first to be produced on line $l$ ( 0 otherwise)

$R N_{l(j, c)}$ equals 1 , if job $(j, c)$ is the last to be produced on line $l$ ( 0 otherwise)

$V_{(i, d)(j, c)}$ equals 1 , if job $(j, c)$ is scheduled right after $(i, d)$ (0 otherwise)

$C t_{(j, c)} \quad$ completion time of job $(j, c)$

The batch scheduling coupled with the vehicle routing problem with time windows (I-BS-VRPTW) may be formulated as follows:

\section{I-BS-VRPTW}

$$
\begin{array}{r}
\min \sum_{l,(i, d),(j, c)} s c b_{l i j} V_{(i, d)(j, c)}+\sum_{l,(j, c)} s c b_{l \alpha_{l}, j} R 0_{l(j, c)}+\sum_{l,(j, c)} c p_{l(j, c)} d e m_{j c} R_{l(j, c)} \\
+\bar{f} t \sum_{k}\left(1-x_{0, n+1}^{k}\right)+\sum_{k} \sum_{c, d} c t_{c d} x_{c d}^{k}
\end{array}
$$

subject to

$$
\begin{gathered}
\sum_{(j, c)} R 0_{l(j, c)} \leq 1 \quad \forall l \\
R 0_{l(j, c)} \leq R_{l(j, c)} \quad \forall l,(j, c) \\
\sum_{(j, c)} R N_{l(j, c)} \leq 1 \quad \forall l \\
R N_{l(j, c)} \leq R_{l(j, c)} \quad \forall l,(j, c)
\end{gathered}
$$




$$
\begin{aligned}
& \sum_{l} R_{l(j, c)}=1 \quad \forall(j, c) \\
& R_{l(i, d)}+V_{(i, d)(j, c)} \leq R_{l(j, c)}+1 \quad \forall l,(i, d),(j, c) \\
& \sum_{l} R 0_{l(j, c)}+\sum_{(i, d)} V_{(i, d)(j, c)}=1 \quad \forall(j, c) \\
& \sum_{l} R N_{(j, c) l}+\sum_{(i, d)} V_{(j, c)(i, d)}=1 \quad \forall(j, c) \\
& C t_{(j, c)} \geq C t_{(i, d)}+\max _{l}\left\{\operatorname{Cap}_{l}\right\}\left(V_{(i, d)(j, c)}-1\right) \\
& +\sum_{l}\left(t p_{l j} d e m_{j c}+s t b_{l i j}\right) R_{l(j, c)} \quad \forall(i, d),(j, c) \\
& C t_{(j, c)} \geq \sum_{l}\left(t p_{l j} d e m_{j c}+s t b_{l \alpha_{l}, j}\right) R 0_{l(j, c)} \quad \forall(j, c) \\
& C t_{(j, c)} \leq \max _{l}\left\{C a p_{l}\right\}+\left(C a p_{l}-\max _{l}\left\{C a p_{l}\right\}\right) R_{l(j, c)} \quad \forall l,(j, c) \\
& f_{c} \geq C t_{(j, c)} \quad \forall(j, c) \\
& C t_{(j, c)}-t p_{l j} d e m_{j c}+s l_{j}-\sum_{k} w_{c}^{k} \geq 0 \quad \forall l,(j, c) \\
& w_{0}^{k} \geq f_{c}-\max _{l}\left\{C a p_{l}\right\}\left(1-\sum_{d} x_{c d}^{k}\right) \quad \forall k, c \\
& \sum_{k} \sum_{d} x_{c d}^{k}=1 \quad \forall c \\
& \sum_{d} x_{0 d}^{k}=1 \quad \forall k \\
& \sum_{c} x_{c d}^{k}-\sum_{c} x_{d c}^{k}=0 \quad \forall k, d
\end{aligned}
$$


Objective function (1) minimizes supply chain related costs, namely: sequence dependent setup costs, variable productions costs, and fixed and variable transportation costs.

Constraints $(2)$ - (6) assign each job $(j, c)$ to a line either in the beginning, in the end or in the middle of the scheduling sequence. Constraints (7) ensure that consecutive jobs are assigned to the same line. Equations (8) establish that a job is either assigned in the beginning of the scheduling or preceded by other job. Similarly, equations (9) impose that a job is assigned at the end of the scheduling or precedes other job. For tracing the completion time of each job, constraints (10) and (11) are used. Note that in (10), $\max _{l}\left\{C_{a p}\right\}$ denotes the latest possible completion time due to the capacity limitations of the lines. Also, these constraints are responsible for the job scheduling. Job completion time must not exceed the available capacity of the line which is assigned (12). Constraints (13) define $f_{c}$, which tracks the customer order finishing time. To account for perishability, (14) assures that the delivery is performed while products still have some lifetime.

In (15) the link between production and the vehicle departing times is established. This synchronization ensures that a vehicle only departs after the completion of the production for all customers visited along the vehicle's route. Constraints (16)-(22) refer to the distribution process. Each customer is visited exactly once by (16), while constraints (17)-(19) ensure that each vehicle is used once and that flow conservation is satisfied at each customer vertex. $x_{0, n+1}^{k}=1$ means that the vehicle was not used. The consistency of the time variables $w_{c}^{k}$ is ensured through constraints (20), while time windows are imposed by (21). Regarding the vehicle capacity, constraints (22) enforce it to be respected. Finally, the domain of the variables is limited by (23). 


\subsection{Integrated Lot Sizing and Scheduling and Vehicle Routing Problem (I- $L S-V R P T W$ )}

Due to production planning modelling reasons, the planning horizon is divided in the lot sizing formulation into a fixed number of non-overlapping slots, indexed by $s$, of variable length. Since the production lines can be independently scheduled, this partition is done for each line separately $(s \in$ $S_{l}$ ). The length of a production slot is a decision variable that is a function of the production quantity of a certain product on a line and of the time to set up the machine for this product (in case it is required). A sequence of consecutive production slots, where the same product is produced on the same line, defines the size of the lot of a product. Therefore, a lot may span over several slots. The number of production slots of a certain line defines the upper bound on the number of setups and deliveries to be performed during the planning horizon.

Contrarily to the more tactical lot sizing and scheduling formulations that integrate the delivery process (Boudia et al., 2007), this model considers a continuous time scale since the external factors, such as demand are pulled from the customer desires, expressed in its time window boundaries. Notice that the slot structure of the mathematical formulation related to the production planning resembles the micro-period time structure of the general lot sizing and scheduling problem (Fleischmann \& Meyr, 1997).

Consider the additional decision variables.

\section{Decision Variables}

$q_{l j s}^{c} \quad$ quantity of product $j$ produced in slot $s$ on line $l$ to serve customer $c$

$y_{l j s} \quad$ equals 1 , if line $l$ is set up for product $j$ in slot $s$ (0 otherwise)

$z_{\text {lijs }}$ equals 1 , if a changeover from product $i$ to product $j$ takes place at the beginning of slot $s$ on line $l$ ( 0 otherwise)

$s t r_{l s} \quad$ starting time of production slot $s$ on line $l$

$\lambda_{l j s}^{c} \quad$ equals 1 , if there is production of product $j$ for customer $c$ in production slot $s$ on line $l$ (0 otherwise)

$F_{j}^{c} \quad$ starting time of the lifespan of product $j$ for customer $c$

The lot sizing and scheduling coupled with the vehicle routing problem with time windows (I-LS-VRPTW) is formulated as follows: 


\section{I-LS-VRPTW}

$\min \sum_{l, i, j, s} s c b_{l i j} z_{l i j s}+\sum_{l, j, s, c} c p_{l j} q_{l j s}^{c}+\bar{f} t \sum_{k}\left(1-x_{0, n+1}^{k}\right)+\sum_{k} \sum_{c, d} c t_{c d} x_{c d}^{k}$

subject to

$$
\begin{gathered}
\sum_{l, s} q_{l j s}^{c}=d e m_{j c} \quad \forall j, c \\
\sum_{c} q_{l j s}^{c} \leq \frac{C a p_{l}}{t p_{l j}} y_{l j s} \quad \forall l, j, s \\
\sum_{j} y_{l j s}=1 \quad \forall l, s \\
y_{l \alpha_{l}, 0}=1 \quad \forall l \\
\sum_{i, j, s} s t b_{l i j} z_{l i j s}+\sum_{j, s, c} t p_{l j} q_{l j s}^{c} \leq C a p_{l} \quad \forall l \\
z_{l i j s} \geq y_{l i, s-1}+y_{l j s}-1 \quad \forall l, i, j, s \\
s t r_{l s} \geq s t r_{l, s-1}+\sum_{i, j} s t b_{l i j} z_{l i j, s-1}+\sum_{j, c} t p_{l j} q_{l j, s-1}^{c} \quad \forall l, s>1 \\
q_{l j s}^{c} \leq d e m_{j c} \lambda_{l j s}^{c} \quad \forall l, j, s, c \\
f_{c} \geq-C a p_{l}\left(1-\sum_{j} \lambda_{l j s}^{c}\right)+s t r_{l s}+\sum_{i, j} s t b_{l i j} z_{l i j s}+\sum_{j, d} t p_{l j} q_{l j s}^{d} \quad \forall l, s, c \\
F_{j}^{c}+s l_{j}-\sum_{k} w_{c}^{k} \geq 0 \quad \forall j, c: d e m_{j c}>0 \\
F_{j}^{c} \leq C a p_{l}\left(1-\lambda_{l j s}^{c}\right)+s t r_{l s}+\sum_{i} s t b_{l i j} z_{l i j s} \quad \forall l, j, s, c
\end{gathered}
$$




$$
q_{l j s}^{c}, z_{l i j s}, s t r_{l s}, f_{c}, F_{j}^{c}, w_{c}^{k} \geq 0 ; y_{l j s}, \lambda_{l j s}^{c}, x_{c d}^{k} \in\{0,1\}
$$

In the objective function (24) the same costs are minimized as in batching related formulation.

Looking now at the constraints that this problem is subject to, demand is to be satisfied with production that may come from different lines (25). Constraints (26) ensure that a product can only be produced if there exists a setup for it and constraints (27) limit to one the number of products to be simultaneously produced on each line. Constraints (28) set the initial configuration of the lines. Limited capacity in the lines is to be used by setup times and the time consumed producing products (29). The connection between setup states and changeover indicators for products is established by (30). In order to define $f_{c}$ that tracks the customer order finishing time in constraint (33), the starting time of each production slot is traced with (31). Requirements (32) determine the customers for which the production in a given slot is devoted to. It is worth mentioning that this production may satisfy demand from several customers. Constraints (34) and (35) account for product perishability similarly to equations (13) and (14). Note, that the model formulation allows for the production of the same product for different customers in a single slot. In such case, $f_{c}$ and $F_{j}^{c}$ are considering only the end and the start of the time slot, therefore, this variables are not considering the exact time of production for each customer. However, this situation can always be avoided by producing the same product of different customer orders in separate (possibly subsequent) slots without additional cost or capacity needs $\left(s c b_{l i i}=s t b_{l i i}=0\right)$. Constraints (15)-(22) from the previous model are also used in this one.

The domain of variables is stated in (36) and the remaining constraints come from the integrated model with batching decisions (I-BS-VRPTW).

\subsection{Relation Between both Models}

The meaning of the main decision variables of both formulations is graphically presented in Figure 1. It is easy to see that both solutions of this illustrative example are equivalent, as the two jobs of I-BS-VRPTW are not split in the I-LS-VRPTW. While the production quantities need to be explicitly tracked in the I-LS-VRPTW, this is not the case for the batching 
model. In terms of setup variables, the two models are very similar, but the lot sizing model has these variables linked to the micro-period, whereas the I-BS-VRPTW uses a continuous representation.

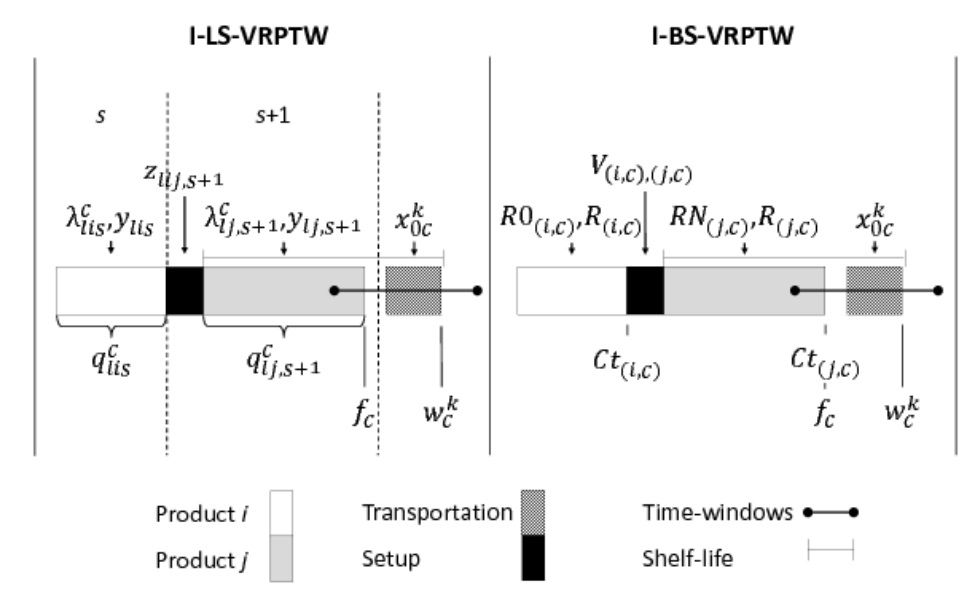

Figure 1: Comparing the decision variables of I-BS-VRPTW and I-LS-VRPTW.

In the following theorem it is shown that the optimal solution to I-LSVRPTW is at least as good as the optimal solution to I-BS-VRPTW. Let $\nu(\cdot)$ denote the optimal values of underlying optimization problems.

Proposition 1. We have $\nu(I-L S-V R P T W) \leq \nu(I-B S-V R P T W)$.

Proof. We prove the statement by showing that I-BS-VRPTW is a special case of I-LS-VRPTW and therefore any feasible solution to I-BS-VRPTW is also feasible to I-LS-VRPTW. Let model $\bar{f}_{L S}$ be derived from I-LS-VRPTW by adding to the latter the following constraints: $\sum_{l, s \in S_{l}} \lambda_{l j s}^{c}=1$, for every $j$ in $N$ and $c$ in $C$, and $\sum_{c, j} \lambda_{l j s}^{c}=1$, for every $l$ and $\mathrm{s}$ in $S_{l}$. These conditions mean that demand for a given pair product $j$-customer $c$ is produced in just one lot, and that each production slot can only be allocated to pair $j-c$. Now, we show the equivalence between I-BS-VRPTW and $\bar{f}_{L S}$. Let $Q^{*}\left(f_{c}, C t_{h}, w_{c}^{k}, R_{l h}, R 0_{l h}, R N_{l h}, V_{h^{\prime} h}, x_{c d}^{k}\right)$ be a feasible solution to I-BS-VRPTW. Each job $h$ entails a product $j$ to be produced and delivered to a customer $c$. Consider in the following a given line $l$. Each job of I-BSVRPTW relates to one production slot of $\bar{f}_{L S}$. The sequence $\left(h_{1}, h_{2}, \ldots, h_{g}\right)$ 
can be easily transformed into the sequence $\left(j_{1}-c_{1}, j_{1}-c_{2}, \ldots, j_{p}-c_{n}\right)$, where the quantity of each product produced in each slot $\left(q_{l j s}^{c}\right)$ equals the amount of demand of the respective job. In case job $h$ in I-BS-VRPTW is produced in the $s$-th position of the sequence, its completion time $\left(C_{h}\right)$ is equivalent in $\bar{f}_{L S}$ to the finishing time of the $s$-th slot where the respective product $j$ is produced to supply the same customer $c$ (i.e. $C_{h}=$ $\left.s t r_{l s}+\sum_{i, j} s t b_{l i j} z_{l i j s}+\sum_{j, c} t p_{l j} q_{l j s}^{c}\right)$. Moreover, the starting time of the lifespan of product $j$ for customer $c\left(F_{j}^{c}\right)$ in $\bar{f}_{L S}$ is equivalent to the term $C_{h}-t p_{l h}$ of the respective job in I-BS-VRPTW. Clearly, $Q$ fulfils the constraints related to the production part of $\bar{f}_{L S}$, from (25) to (34). The routing-related requirements are the same in both formulations. This clearly shows that $\nu(\mathrm{I}-\mathrm{LS}-\mathrm{VRPTW}) \leq \nu\left(\bar{f}_{L S}\right) \leq \nu(\mathrm{I}-\mathrm{BS}-\mathrm{VRPTW})$.

\section{Computational Study}

This section aims at quantifying the impact of considering lot sizing versus batching and analysing the solution changes that this extra production flexibility yields. To this end, a set of instances have been systematically generated with different parameters. Next it is reported how the test instances are generated. Afterwards, the computational results are presented and, finally, some examples comparing the improvements of the lot sizing over the batching solutions are analysed.

\subsection{Data Generation}

The instance generators used by Haase \& Kimms (2000), Armstrong et al. (2007) and Viergutz (2011) are integrated since, to the best of our knowledge, there are no reported instances for the settings of this problem. A total of 120 instances were generated. The impact of different key parameters on the lot sizing importance is verified by varying: the number of perishable products, the length of the shelf-life, the setup time and cost structure, the tightness of the time windows and the orientation of the time windows.

For the sake of compactness, the description of parameters' generation is exposed only for I-LS-VRPTW. However, the data conversion for I-BSVRPTW is straightforward. The number of lines $m$ is set to 1 and for all products $t p_{l j}=1$ and $c p_{l j}=0$. In the beginning of the planning horizon the machine is set up for product 1 . There are 3 items $(p=3)$ to be produced for 5 customers $(n=5)$. The number of production slots $S_{l}$ is set to $p \times n$ in order to ensure that all necessary setups and deliveries may be performed. 
$75 \%$ of the demand $d e m_{j c}$ is generated from the uniform distribution in the interval $U[40,60]$ and the remaining $25 \%$ is set to 0 . The number of perishable products $(P P)$ can be 1 or 2 out of 3 items. In order to define the length of the shelf-life of perishable products $\left(s l_{j}\right)$, parameter $S L$ is multiplied by the average quantity of a demand order. This parameter $S L$ can be 3 or 5 .

The setup time and cost structure may obey or not to the triangular inequality. In case setups obey to triangular inequality, in the optimal solution the production of the same product will never take place twice in the same period. On the contrary, setups not obeying to the triangular inequality, which are frequent in the food industry with the use of cleaning products, may result in optimal solutions in which the same product is set up more than once in the same period (favoring the lot sizing). For the instances with triangular setup times $(T S)$ between products $s t b_{l i j}, U[6,10]$ is used for all pairs (except for the case where $i=j$, where the setup is 0 ). The instances not obeying to such inequality $(N T S)$ have setup times chosen randomly from $U[1,5]$. The setup costs $s c b_{l i j}$ of a changeover from product $i$ to $j$ are computed as:

$$
s c b_{l i j}=25.0 \cdot s t b_{l i j} \text { and } s c b_{l i j}=66.67 \cdot s t b_{l i j},
$$

for triangular and non-triangular setups, respectively. Both setup structures have an average setup cost of 200 units. The line capacity $\mathrm{Cap}_{l}$ is determined according to:

$$
C a p_{l}=\frac{\sum_{j c} d e m_{j c} t p_{l j}}{0.6} .
$$

It is important to notice that the capacity utilization (0.6) is an estimate only, as setup times do not influence the computation of $\mathrm{Cap}_{l}$. We also enforce mininum batch sizes to be equal to the smaller order quantity, therefore, this condition only influences the lot sizing model in order not to perform very small production lots.

For the computation of the travel times $t t_{c d}$ and costs $c t_{c d}$, which are assumed to be the same, all customers are positioned randomly in a square of locations from $(0,0)$ to $(100,100)$. The Euclidean distance is then calculated between all pairs of customers (assuming that travel times are equal to the travel distances) fixing the depot at the point $(50,50)$. The service times $s_{c}$ are negligible. The number of available vehicles is set to $n$ (number of customers) and the cost of using each vehicle $\bar{f} t$ is set to 250 . This value was set after preliminary computational experiments to reflect the industry 
practice in relation to the vehicle variable costs. The capacity of the vehicle is computed through the expression

$$
C a p V=0.5 \sum_{j c} d e m_{j c} .
$$

For all customers, no service time $\left(s_{c}\right)$ necessary. The last parameters are the time windows of each customer (parameters $a_{c}$ and $b_{c}$ ), which are calculated by four different methods that change the tightness and the orientation of the time windows. These methods are described in Appendix A. With regard to the tightness, instances with standard $(S)$ and loose $(L)$ time windows are considered. Concerning their orientation, instances with time windows oriented by production requirements $(P)$ and by customers' demand $(C)$ are considered.

By varying the aforementioned parameters, 24 types of instances are generated. For each of them, 5 random instances are considered. All the 120 instances were tested for feasibility purposes on the I-BS-VRPTW model with a commercial solver. In case a solution had not be found, then a new instance was generated until feasibility was achieved.

\subsection{Computational Results}

All computational experiments were performed on an HP Z800 workstation with two six-core Intel Xeon X5690 at $3.47 \mathrm{GHz}$ with 48 GB RAM, running Linux. CPLEX version 12.4 from IBM was used as the MIP solver. The data generator described in Section 4.1 was used to obtain the instance set. The computational time to solve each MIP is limited to 3600 seconds. As the I-BS-VRPTW was solved to optimality by CPLEX within a maximum/average running time of $126.97 / 6.07$ seconds, these solutions were used as a starting point for the I-LS-VRPTW (i.e. they were injected into its branch-and-bound tree).

In order to evaluate the solutions of the two models we use indicator gap $_{\text {sol }}$, that refers to the relative difference of solutions between the I-LSVRPTW $\left(U B_{L}\right)$ and I-BS-VRPTW $\left(U B_{B}\right)$. These gap is calculated as:

$$
\operatorname{gap}_{\text {sol }}=\frac{U B_{B}-U B_{L}}{U B_{L}} .
$$

Table 1 provides the solution improvement gap $_{\text {sol }}$ of I-LS-VRPTW over I-BS-VRPTW for the all the instances. Here, The sign "-" means that the IBS-VRP-TW solution was not improved by I-LS-VRP-TW model, within the 
time limit. Notice that the average integrality gap of the lot sizing solutions is $6.3 \%$ The cause behind the solution improvements is also presented in the same table. In general, the cost decrease on the solution of I-LS-VRPTW may yield five main changes in relation to the solution of I-BS-VRPTW:

- St- $(+)$ : number of setup operations;

- Sc- $(+)$ : total setup cost;

- Seq: setup sequence;

- Dist- $(+)$ : distance travelled;

- $\mathrm{V}-(+)$ : number of used vehicles.

The signs - $(+)$ mean a decrease (increase) of the indicator of the respective change. Notice that, contrarily to the case of triangular setup structure, the case of non-triangular setups may allow for setup inclusions $(\mathrm{St}+)$ that result in setup cost reduction (Sc-). Therefore, Sc- is omitted for triangular setups when the related changes are due to St- or Seq.

I-LS-VRPTW obtained better solutions for 35 out of 120 instances. In 22 instances both formulations reported the same provably optimal solution and there are 40 instances for which it is still theoretical possible to improve the batching solution by allowing for lot size. The maximum gap $_{\text {sol }}$ is $20.0 \%$ caused by the reduction of setup operations. The average $g a p_{s o l}$, for instances with positive gaps, is $6.5 \%$. The main cause of cost decrease, when lot sizing is allowed, is the reduction of setup operations, which was responsible for 21 out of the 35 instances improved. Customer oriented time windows $(C)$ has leveraged the number of solutions improved by lot sizing. Loose time windows $(L)$ allowed more changes related to distribution decisions. Moreover, nontriangular setups (NTS) increased the number of instances improved by I-LS-VRPTW.

\subsection{Solution Examples}

In this subsection, illustrative examples of instances in which the I-LSVRPTW overcomes the I-BS-VRPTW are shown. In each example, two Gantt charts are used to represent graphically the solutions. The top chart represents the Gantt chart of the I-BS-VRPTW solution and the bottom illustrates the I-LS-VRPTW solution. Customers are arranged according to their time windows boundaries and vertically at the Gantt chart, from 
Table 1: Gaps between batching and lot sizing solutions.

customer 1 to 5. Products 1, 2 and 3 are denoted by light grey, dark grey and dotted bars, respectively. Setup operations are in black colour bars. The shelf-lives of perishable products are represented by thin white bars starting at the beginning of the production process. The time windows boundaries are indicated by two vertical lines delimiting delivery operations. The travel time from the depot (or customer) to a customer is represented by 45 degree downward hatch box and the opposite operation, from customer to depot, by a 45 degree upward hatch bar. Moreover, the jobs that were split are pointed out by an upward or a downward arrow in the respective I-LS-VRPTW graphical solution.

In example 1 of Figure 2 lot sizing can improve the solution of I-BSVRPTW by reducing setup operations (St-). In the I-BS-VRPTW solution the setup sequence is $(1,2,1,2,3,1,3,2)$. With the lot sizing flexibility, it is possible to better use the shelf-life limitation of product 2 for customer 2 and rearrange the production sequence by sizing the lot of product 1 for customer 2. Thus, the new setup sequence is $(1,2,1,3,2,1)$, which entails two less setup operations, one for product 2 and one for product 3 . The delivery operations are the same for both solutions.

Example 2 (Figure 3) is similar to example 1, but instead of reducing 


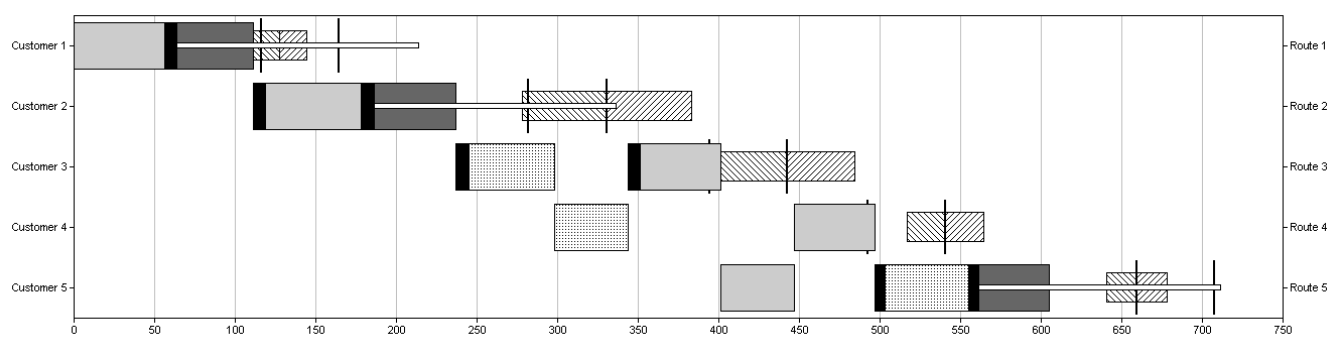

(a) I-BS-VRPTW

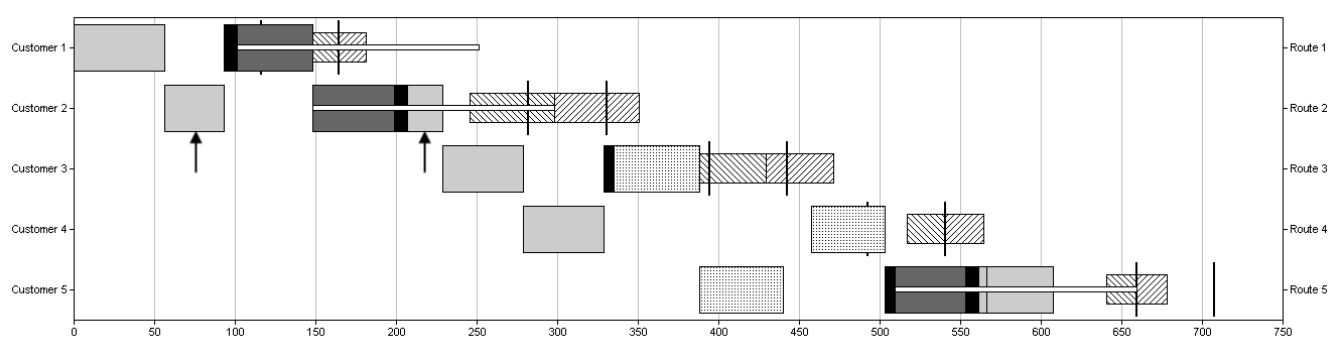

(b) I-LS-VRPTW

$$
\begin{aligned}
& \square \text { Item } 1 \quad \square \text { Item } 2 \quad \square \text { Item } 3 \quad \square \text { Setup } \square \text { Shelf Life } \\
& \text { || Time Window Boundaries } \quad \square \text { Vehicles } \square \text { Vehicles Back }
\end{aligned}
$$

Figure 2: I-BS-VRPTW and I-LS-VRPTW solutions to instance $\mathrm{PP}=1, \mathrm{SL}=3, \quad \#=4$, C-S-TS (St-). 
the number of setup operations, lot sizing has enabled a modification of the setup sequence (Seq), resulting in a lower solution cost. This example shows the importance that lot sizing can have when setup costs do not obey to the triangular inequality. It is noticeable that the changeover from product 1 to 2 is more economic if product 3 is produced in between. The lot sizing operation allows for such setup sequence, while the products are still delivered without getting spoiled. Moreover, by sizing the lot of product 1 for customer 2 it was possible to reduce one setup for product 2 .

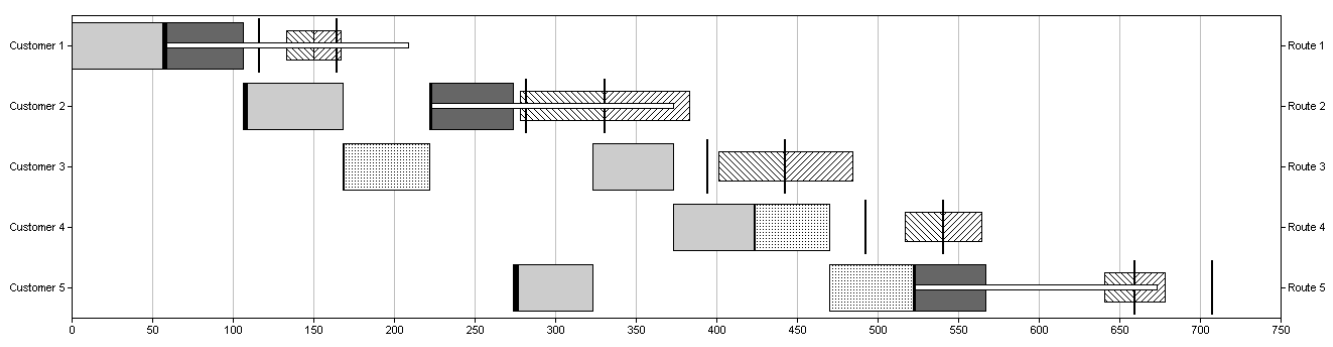

(a) I-BS-VRPTW

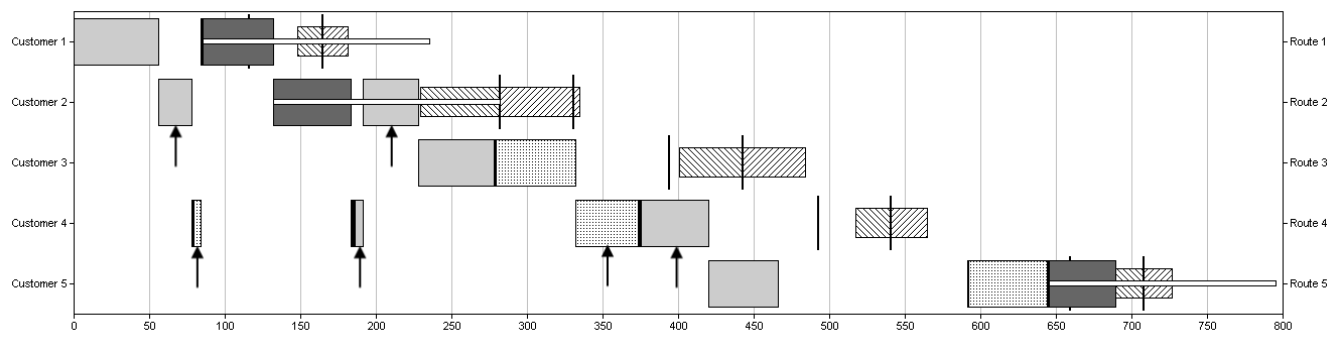

(b) I-LS-VRPTW

Figure 3: I-BS-VRPTW and I-LS-VRPTW solutions to instance PP=1, SL=3, \#=4, C-S-NTS (Seq).

In the example of Figure 4, the difference between batching and lot sizing solutions is once again related to the reduction of setups. However, in this case, the delivery operations were also changed (Dist+, St-). The splitting of job $(3,3)$ - product 3 for customer 3 - allowed a single batch production of product 2. This production change yields a different routing maintaining the same number of vehicles. Hence, the reduction of the setup costs counterweights the increase of the distance travelled. 


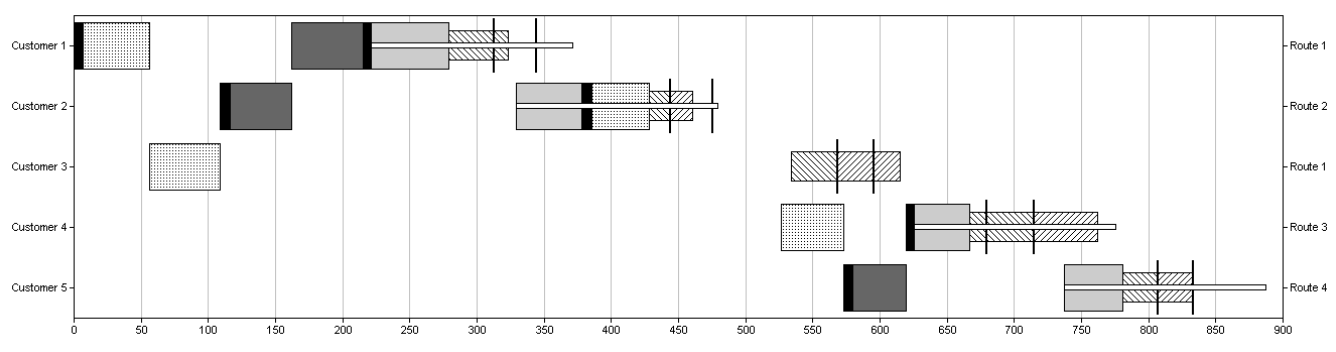

(a) I-BS-VRPTW

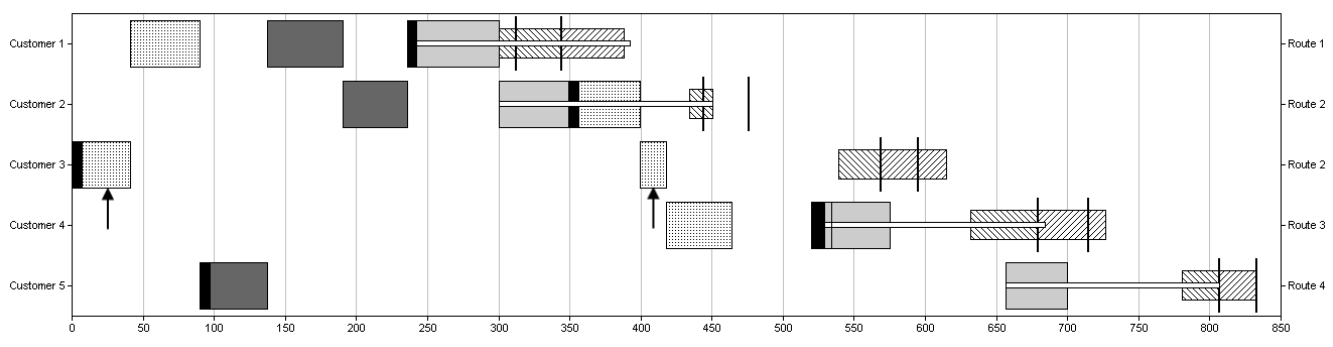

(b) I-LS-VRPTW

Figure 4: I-BS-VRPTW and I-LS-VRPTW solutions to instance $\mathrm{PP}=1, \mathrm{SL}=3, \#=5$, P-L-TS (Dist+, St-). 
Figure 5 shows an instance where the travel costs decrease due to the routing change provided by lot sizing (Dist-) and the setup costs remain unchanged. The batching solution uses a vehicle for supplying customers 1 and 4 and another for customers 2 and 3. When lot sizing is allowed, customers 1 and 3 are part of the same vehicle's route while customers 2 and 4 belong to other.

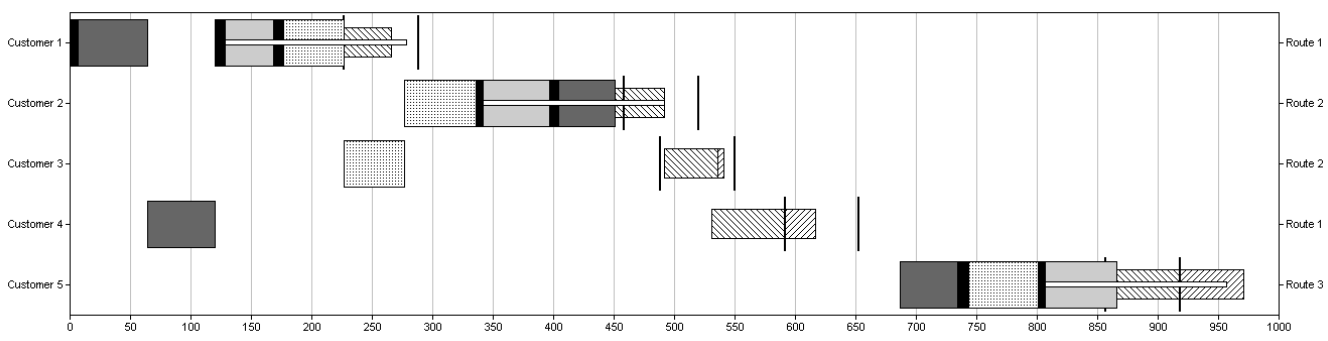

(a) I-BS-VRPTW

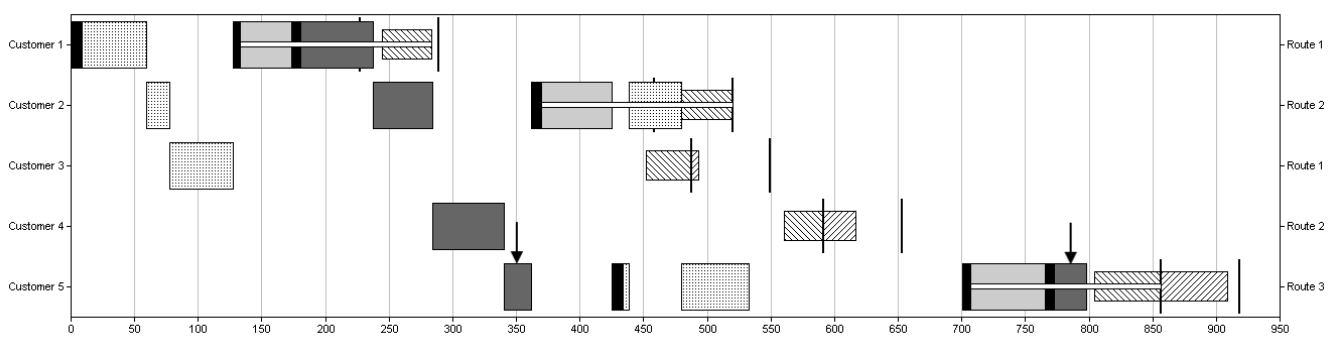

(b) I-LS-VRPTW

Figure 5: I-BS-VRPTW and I-LS-VRPTW solutions to instance $\mathrm{PP}=1, \mathrm{SL}=3, \#=2$, C-L-TS (Dist-).

Finally, Figure 6 illustrates the improvement of a batching solution by means of the reduction of one vehicle (V-, Dist-, St+). With the splitting of job $(1,2)$ - product 1 for customer 2 , it is possible to serve customers 1 and 4 along the same route. It is interesting to note that in this solution, the usage of customers' time windows up to the boundary. In the batching solution, only customers 3 and 4 share a vehicle's route, while all the others are supplied by different vehicles. On the other hand, the lot sizing solution only uses three vehicles, also reducing the travel costs. However, more setup operations are needed increasing the total setup costs (that does not surpass 
the distribution costs decrease). In the batching solution, the setup sequence is $(1,2,3,2)$, against $(1,3,2,1,3,2)$ of the lot sizing model.

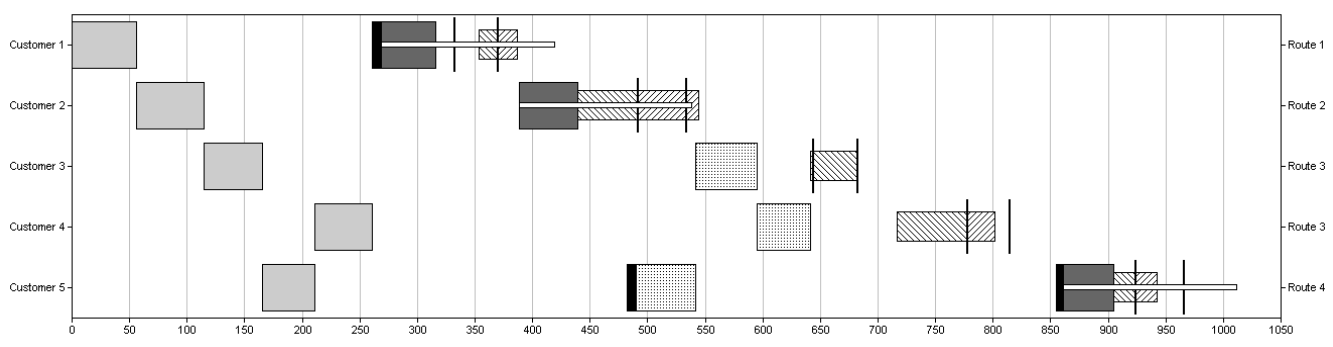

(a) I-BS-VRPTW

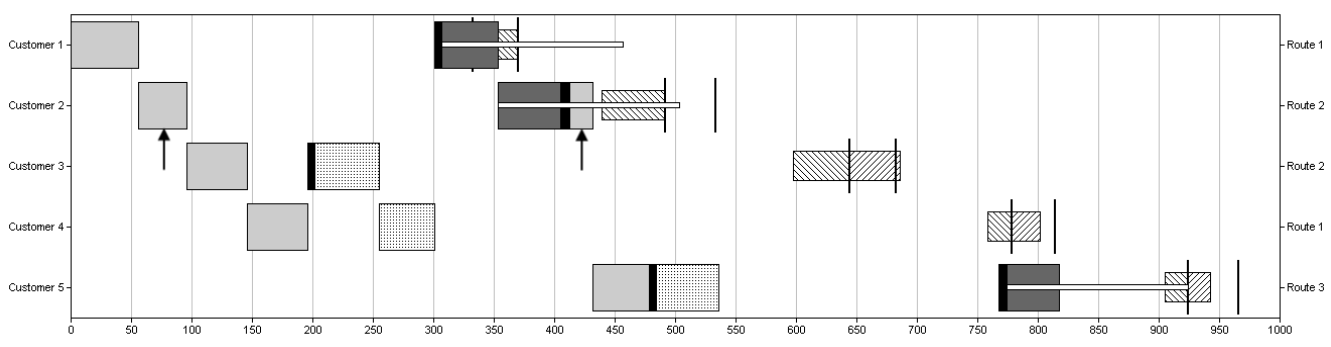

(b) I-LS-VRPTW

Figure 6: I-BS-VRPTW and I-LS-VRPTW solutions to instance $\mathrm{PP}=1, \mathrm{SL}=3$, $\#=4$, P-L-TS (V-, Dist-, St+).

\section{Conclusions}

In this paper, we have analysed the importance of considering sizing the lots (or in other words, splitting the jobs) besides pure batching at the operational production and distribution planning when considering perishability. The lot sizing decision is a counterintuitive one in make-to-order environments and this is the first time that its importance is analysed. The logistic setting of our operational problem encompasses multiple perishable products subject to sequence dependent changeovers, which have to be delivered in a certain route by one of the available vehicles. We have developed models for accurately integrate both lot sizing and batching with the vehicle routing problem with time windows. In order to understand the impact of the 
extra flexibility coming from the possibility of splitting the lots, experiments varying different key parameters are designed and the solutions between the batching and lot sizing models are compared.

Computational results for the set of systematically generated instances show that lot sizing is able to decrease the integrated production and distribution costs on very different types of instances. Both customer oriented time windows and production environments with non-triangular setups seem to favour the importance of considering lot sizing in this operational problem. Several mechanisms to improve the batching solution were found by the lot sizing model. The lot sizing solution could achieve a better performance by: reducing the number of setups, changing the sequence, reducing setup costs, reducing the number of vehicles and/or the total travelled distance.

\section{References}

Almada-Lobo, B., Klabjan, D., Carravilla, M. A., \& Oliveira, J. F. (2010). Multiple machine continuous setup lotsizing with sequence-dependent setups. Computational Optimization and Applications, 47, 529-552.

Armstrong, R., Gao, S., \& Lei, L. (2007). A zero-inventory production and distribution problem with a fixed customer sequence. Annals of Operations Research, 159, 395-414.

Boudia, M., Louly, M., \& Prins, C. (2007). A reactive GRASP and path relinking for a combined productiondistribution problem. Computers $\&$ Operations Research, 34, 3402-3419.

Chen, H.-K., Hsueh, C.-F., \& Chang, M.-S. (2009). Production scheduling and vehicle routing with time windows for perishable food products. Computers \& Operations Research, 36, 2311-2319.

Chen, Z.-L. (2009). Integrated Production and Outbound Distribution Scheduling: Review and Extensions. Operations Research, 58, 130-148.

Chen, Z.-L., \& Vairaktarakis, G. L. (2005). Integrated Scheduling of Production and Distribution Operations. Management Science, 51, 614-628.

Fleischmann, B., \& Meyr, H. (1997). GLSP. OR Spektrum, 19, 11-21. 
Geismar, H. N., Laporte, G., Lei, L., \& Sriskandarajah, C. (2008). The Integrated Production and Transportation Scheduling Problem for a Product with a Short Lifespan. INFORMS Journal on Computing, 20, 21-33.

Haase, K., \& Kimms, A. (2000). Lot sizing and scheduling with sequencedependent setup costs and times and efficient rescheduling opportunities. International Journal of Production Economics, 66, 159-169.

Low, C., \& Yeh, Y. (2008). The impact of lot splitting in a single machine scheduling problem with earliness - tardiness penalties. In M. Mitsuishi, K. Ueda, \& F. Kimura (Eds.), Manufacturing Systems and Technologies for the New Frontier (pp. 293-296). Springer London.

Mendez, C., Henning, G., \& Cerda, J. (2000). Optimal scheduling of batch plants satisfying multiple product orders with different due-dates. Computers 85 Chemical Engineering, 24, 2223-2245.

Nieuwenhuyse, I. V., \& Vandaele, N. (2006). The impact of delivery lot splitting on delivery reliability in a two-stage supply chain. International Journal of Production Economics, 104, 694-708.

Park, Y. B. (2005). An integrated approach for production and distribution planning in supply chain management. International Journal of Production Research , 43, 1205-1224.

Potts, C. N., \& Van Wassenhove, L. N. (1992). Integrating Scheduling with Batching and Lot-Sizing: A Review of Algorithms and Complexity. The Journal of the Operational Research Society, 43, 395-406.

Santos, C., \& Magazine, M. (1985). Batching in single operation manufacturing systems. Operations Research Letters, 4, 99-103.

Sarmiento, A. M., \& Nagi, R. (1999). A review of integrated analysis of production-distribution systems. IIE Transactions, 31, 1061-1074.

Viergutz, C. (2011). Integrated Production and Distribution Scheduling. Ph.D. thesis Universität Osnabrück.

Wagner, B. J., \& Ragatz, G. L. (1994). The impact of lot splitting on due date performance. Journal of Operations Management, 12, 13-25. 


\section{Appendix A: Time Windows Generation}

For the generation of time-windows data, an auxiliary parameter $\tau$ (that estimates the length of a vehicle tour) needs to be defined in two steps. First, a greedy nearest neighbourhood procedure finds a path for all customers without considering time windows. The distance of the solution found is then multiplied by 0.5 in order to account for the necessary expected vehicles (recall that a vehicle is able to carry half of the total demand), defining $\tau$. Let us now define $\mu_{t w}$ as the mean width of the time windows that equals to $0.1 \tau$. Two different methods are responsible for varying the orientation of time windows: production $(P)$ or customer $(C)$ oriented. To generate these time windows the algorithm proposed in Viergutz (2011) is adapted and described in Algorithm 1.

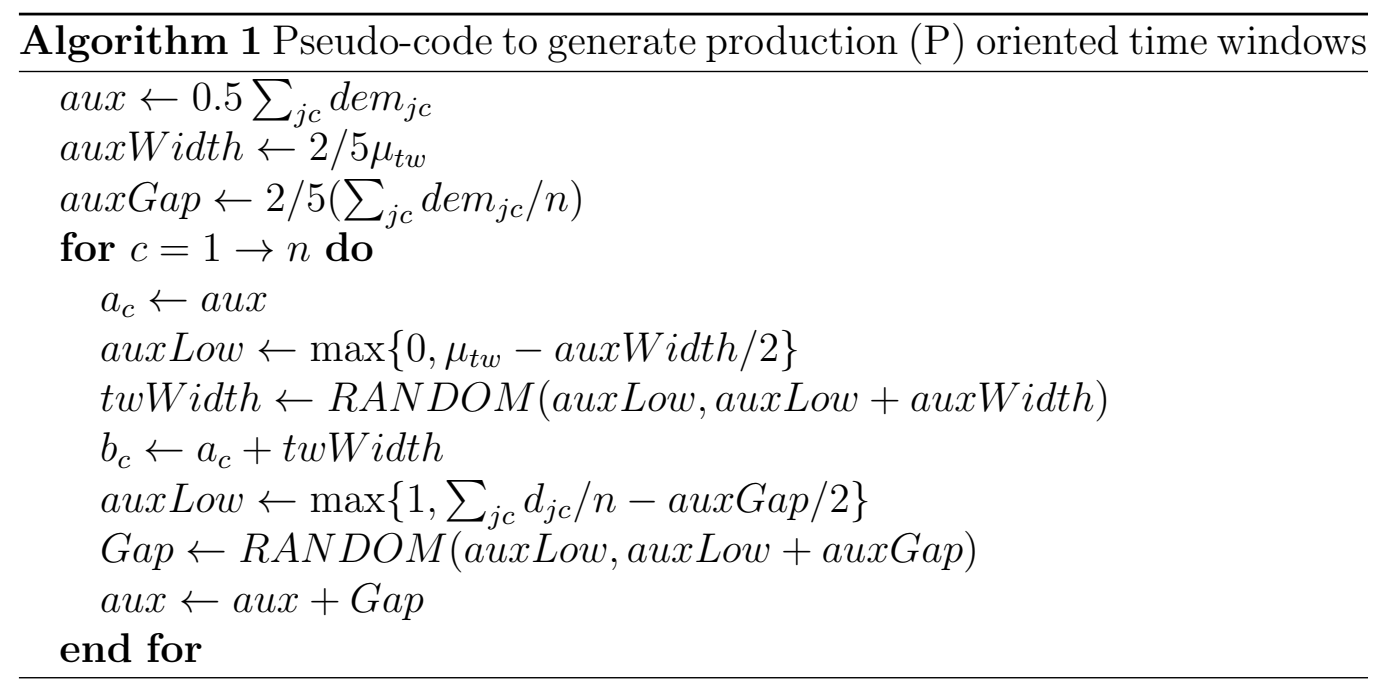

The customer's time windows generated by Algorithm 1 are production $(P)$ oriented, in the sense that the first time window just starts after the necessary time to complete half of the total demand. The second method generates customer $(C)$ oriented time windows and yields a profile in which parameters $a_{c}$ and $b_{c}$ are now defined according to the demand of each customer. Algorithm 2 describes the generator of time windows instances $C$. In the description, the maximum setup time is denoted by maxStb and the value of the average demand element by avDem.

In order to vary the tightness of time windows, the standard $(S)$ tightness of the time windows calculated in Algorithms 1 and 2 is relaxed to achieve 


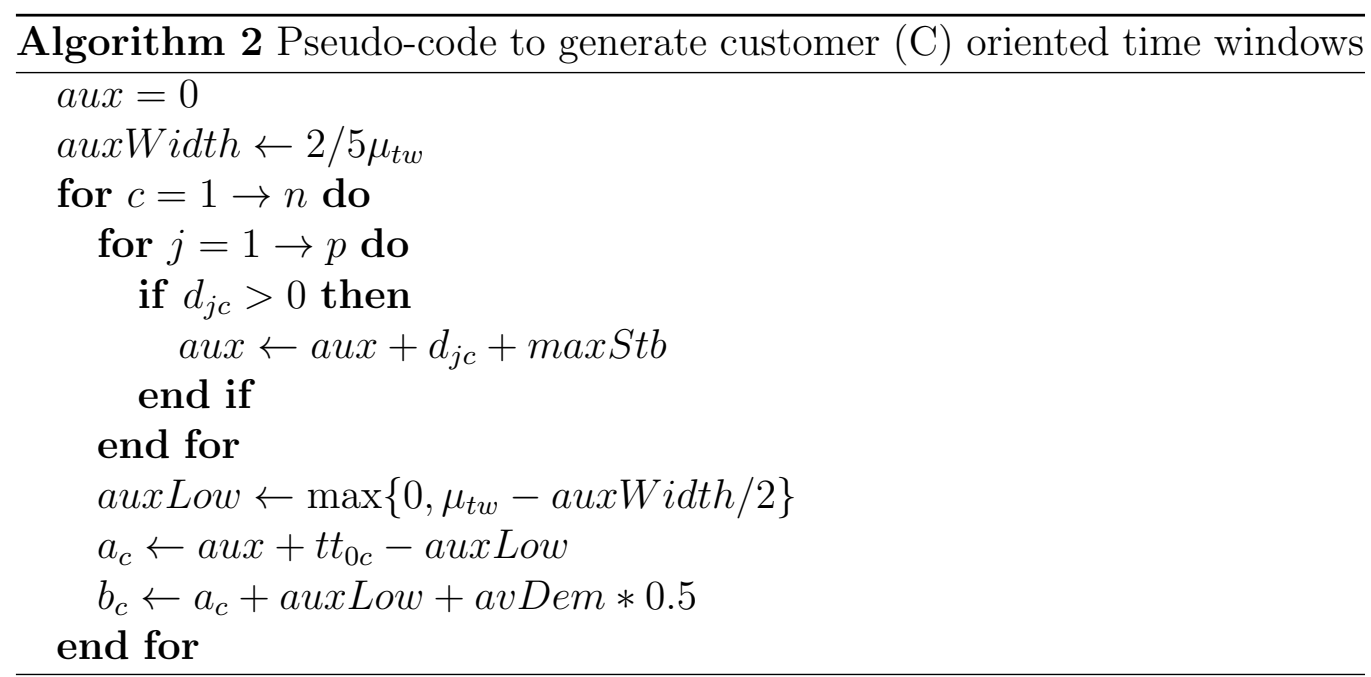

loose $(L)$ time windows. Hence, the $\mathrm{L}$ time windows are calculated by postponing by $20 \%$ the time windows calculated with the previous two methods. Consequently four different types of time windows may be generated, considering the tightness and the orientation of the time windows. 